IMPLANT DENTISTRY; ORTHODONTICS

\section{Orthodontic aspects of the use of oral implants in adolescents: a 10-year follow-up study}

Thilander B, Ödman J et al. Eur J Orthod 2001; 23: 715-731

Different problems occur in the incisor and premolar areas.

This study reports on 15 patients given 29 single implants for aplasia and 3 patients with extensive aplasia given 18 implants; all subjects were aged 13-17 yrs. No implants were lost and aesthetics were considered acceptable throughout the $10 \mathrm{yrs}$.

In upper incisor regions, 7 of 17 single implants became infraoccluded by $0.6-1.6 \mathrm{~mm}$ within $3 \mathrm{yrs}$, and mean infraocclusion for all 17 was $0.98 \mathrm{~mm}$ by $10 \mathrm{yrs}$. The corresponding $3 \mathrm{yr}$ result for 9 premolar implants was 0.1-0.6 mm, but by 10 yrs all crowns were in occlusion.

Implant bone loss was minimal and was greater with a shorter distance to the adjacent tooth. The greatest problems occurred with lateral incisors and were linked by the authors to continuous eruption of adjacent teeth and post-adolescent craniofacial changes. They viewed implant therapy as a good option in extensive aplasia, but facial growth should be complete or nearly so.

\section{ORAL MEDICINE}

\section{Oral manifestations of an HIV positive} cohort in the era of highly active antiretroviral therapy (HAART) in south London

Eyeson JD, Tenant-Flowers M et al. J Oral Pathol Med 2002; 31: 169-174

Most oral lesions were found in patients with high HIV-1 RNA viral loads and low CD4 counts.

In 203 adult subjects (1/4 female, 2/5 heterosexual, 1/4 smokers; mean age $37 \mathrm{yrs})$, half had no oral lesions. The commonest lesions were hairy leukoplakia (HL), HIV-associated periodontal disorders (linear gingival erythema, necrotizing gingivitis and periodontitis) and candidiasis. Subjects with a detectable plasma RNA viral load accounted for most of the leukoplakia and candidiasis.

In $70 \%$ of subjects, 3 drugs were prescribed (reverse transcriptase inhibitors, protease inhibitors) and in 25\%, no therapy was given; the remainder were not on 3 drugs. A surprisingly large number (1/4) had recurrent oral ulceration. Regression analysis suggested only one association with a demographic factor: HIVassociated periodontal disorders were associated with tobacco smoking. The authors considered HL to be low in this population, and attributed this and the overall low prevalence of oral lesions to HAART. Reduced frequency of candidiasis probably also relates to this therapy.
ONCOLOGY

\section{Continuous selective intraarterial chemotherapy in combination with irradiation for locally advanced cancer of the tongue and tongue base}

Furutani K, Fuwa N et al. Oral Oncol 2002; 38: 145-152

In patients who could not undergo the preferred treatment of surgery, combination therapy showed some promise.

Extensive surgery is the usual treatment for cancer of the tongue. In 33 patients of median age 68 yrs with tongue tumours and 6 with tongue base tumours (T2+N0-2M0), who refused surgery or could not be treated surgically for medical reasons, lingual artery chemotherapy and concurrent radiotherapy were given. In 13 with cervical node metastasis, systemic chemotherapy was also given. In 2 patients, chemotherapy could not be completed.

Follow-up was from 9-95 months. At 3 yrs, the local control rate was $88 \%$ for new cases and 50\% for recurrent cases; progression-free survival was 53\%, and overall survival was 59\%. Recurrence in the neck or at distant sites was about 30\%. The authors consider these results might be improved upon and are currently performing a study of an enhanced combination therapy.

\section{ORAL PATHOLOGY}

\section{Cementoblastoma: an innocuous} neoplasm? A clinicopathologic study of 44 cases and review of the literature with special emphasis on recurrence

Brannon RB, Fowler CB et al. Oral Surg 2002; 93: 311-320

Over 1/3 cases recurred within 4-24 months.

Most published data on cementoblastoma are limited by minimal follow-up information. In this study, a retrospective analysis of 44 cases of cementoblastoma (WHO criteria) from the files of the US Armed Forces Institute of Pathology (AFIP) from 1955 to 1980 was compared with 74 cases reported in the clinical literature. In the AFIP cases, the commonest initial finding was swelling with pain, or one of these complaints (28 cases).

In 35 AFIP cases, follow-up was from 4 months to 19 yrs (mean $5.5 \mathrm{yrs}$ ). In 13 of these there was a recurrence within 2 yrs. A literature search (LS) revealed 74 cases which could be verified as cementoblastoma.

In demographic and diagnostic data, the AFIP and LS cases were similar. However, follow-up of LS was a mean 3.4 yrs (range 0.3-11) and the reported recurrence rate was $6 \%$ as opposed to $37 \%$ for AFIP. The authors conclude that the recurrence rate is much higher than previously reported and follow-up should be thorough. They also consider recurrence to be dependent mostly on incomplete removal. 